Modelling vocal imitation

Vocal imitation can create acoustic attractors to guide mothers to pups in a crowded colony of Mexican free-tailed bats: A case-study of computational modelling in behavioural biology

\author{
Richard Shillcock*†, Beren Millidge $\ddagger$, Andrea Ravignani $\S^{* *}$
}

B.M. was supported by EPSRC (UK) grant EP/N509644/1. A.R. was supported by funding from the European Union's Horizon 2020 research and innovation programme under the Marie Skłodowska-Curie grant agreement No 665501 with the Research Foundation Flanders (FWO) (Pegasus2 Marie Curie fellowship $12 \mathrm{~N} 5517 \mathrm{~N}$ awarded to AR).

Corresponding author: rcs@inf.ed.ac.uk tel: +44 1316504425

* School of Philosophy, Psychology and Language Sciences, University of Edinburgh, UK

$\uparrow$ School of Informatics, University of Edinburgh, UK

$¥$ School of Informatics, University of Edinburgh, UK

$\S$ Research Department, Sealcentre Pieterburen, Pieterburen, Netherlands

** Artificial Intelligence Lab, Vrije Universiteit Brussel, Brussels, Belgium 
Modelling vocal imitation

\begin{abstract}
How can we understand vocal imitation, the rare ability of certain species to copy vocalizations and other environmental sounds? How can computational modelling assist us? Here we describe a stepby-step process of mutually accommodating biological data to an implemented computational model. We begin with observations of harbour seals and with a putative computational model of their vocalizations in a colony. At each point in the development of the model, we analyse our decisions from the perspective of a materialist theory of knowledge, drawing on its explicit claims regarding abstraction and the concepts of the abstract universal, the concrete universal and the biological totality. We are eventually led to focus on bats, specifically the Mexican free-tailed bat (Tadarida brasiliensis mexicana). A large colony is a pandemonium of visual, olfactory and auditory cues to a pup's location. Our computational model shows that if each pup's vocalizations are influenced by its neighbours, robust attractors develop in the soundscape across the colony. Vocal imitation radically simplifies the problem of a returning mother finding a particular pup. She need only ascend the gradient of similarity with her own infant's vocalization. This strategy outperforms other simple spatial search strategies and yields a parsimonious explanation for the role of vocal imitation in bats. We reach this modelling conclusion in a principled and transparent manner.
\end{abstract}

Keywords: vocal imitation; bats; computational modelling; parenting behaviour; theory of knowledge; abstraction 
Modelling vocal imitation

Vocal imitation can create acoustic attractors to guide mothers to pups in a crowded colony of Mexican free-tailed bats: A case-study of computational modelling in behavioural biology Computational modelling has emerged in recent decades as a major research paradigm across the sciences. It gives researchers a means of generating proof-of-concept analogies with real systems, exploring emergent effects, rigorously testing hypotheses, and producing a virtuous spiral of modelling and empirical experimentation/observation (cf. McClelland, 2009; Weisberg, 2012). It marries cutting-edge technology and machine-learning algorithms with longstanding philosophical issues concerning abstraction. However, many researchers are not explicit about the process by which their model was developed, leading to ambiguity about the status of computational models and to contrasting theoretical positions (cf. Frigg \& Hartmann, 2018; Weisberg, 2012). In particular, the process of abstraction and the role of universals in the theory of knowledge behind the modelling process are typically left implicit, such that the models are often seen as stand-alone 'explanations' of the real-world phenomena. Computational modelling is by its nature interdisciplinary, requiring the modelling theorist to understand more of the experimental context and forcing the experimentalist to engage with the mathematics and philosophy of modelling.

In the current case study, we explain how we develop the fit between a biological behaviour and a computational model and how the relation between the two affects our understanding of the biological data. At each stage we discuss the decisions taken, drawing metatheoretical conclusions about computational modelling in biological research. We assume a materialist theory of knowledge.

The particular biological data we have eventually targeted is what has been termed vocal imitation in bats, with particular reference to the Mexican free-tailed bat (Tadarida brasiliensis mexicana). However, we begin with the earlier process of homing in on the relevant real-world data. 
Modelling vocal imitation

\section{Identifying the biological 'data'}

Data from an experiment always implies a theory. Seeing a naturalistic observation as 'data' similarly implies a theory. In the current case, the initial biological observation concerned apparent 'vocal imitation' between two juvenile harbour seals (Ravignani, Garcia, Gross, de Reus, Hoeksema, Rubio-Garcia \& de Boer, 2018) and the framing of the questions 'What might be the utility of infant vocal imitation in the harbour seal and how does it come to exist?'

At this point, two directions of travel are possible: towards greater generality (generalization across species) or towards greater specificity (more biological detail, within the species).

One move towards greater generality is already implicit in identifying the phenomenon as 'vocal imitation'. We have categorized the phenomenon as the intersection of the set of vocal behaviours and the set of imitation behaviours, both sets applying across species. Below, we will explore this type of abstraction — an 'abstract universal' - but for now we can note that the term 'vocal imitation' is already a model of sorts, attempting to capture that which is similar across a rather constrained set of animal lineages. In observing two harbour seals, the researcher is 'spontaneously materialist'; i.e. in contact with the rich biological totality of the situation. Any statement about that situation implies abstraction and has profound consequences for the modelling process.

The move towards greater specificity is to consider (at least some of) the fuller context. Harbour seals sometimes live in colonies, meaning that an infant seal hears many different vocalizations from different individuals. What is the nature of vocal imitation within a cacophony of different vocalizations?

Computational modelling comes into its own with such questions. A computational model can orchestrate complex interactions between large numbers of individual entities and reveal surprising emergent effects that are beyond the initial intuitions of the researcher; see, for instance, 
Modelling vocal imitation

the 'artificial life' modelling of flocking behaviour in large numbers of birds (Reynolds 1987), or the simulation of the evolution of plasticity in learning by generations of simple artificial neural network systems (Bullinaria, 2009), or the modelling of the interaction of large numbers of representations of words in speech perception (McClelland \& Elman, 1986). At this point, we have taken the computational models that researchers have constructed to model interaction/ communication between large numbers of individuals, with their interactions organized within simple parameters, and we have generated another 'abstract universal' — 'computational model'capturing that which is similar between those models, and we have proposed that there can be just such a model that captures our current biological data on harbour seals. In such a model, an 'input' or starting condition is specified and the model resolves the ensuing interactions deterministically and its behaviours are recorded. We now describe the instantiation of such a model.

\section{Simulating vocal imitation}

The model consists of a static grid of 50 x 50 individuals. Each individual begins with a random 'vocalization'. To represent acoustic features commonly used in individual recognition, such as fundamental frequency, formant dispersion, and call duration, we employ three real-valued arbitrary 'features' between 0 and 255, represented as colours in the standard RGB colour space (electronic supplementary Fig. ES 1a). At every iteration of the model, each individual alters its vocalization to be the mean of the surrounding calls within a radius $r$. This radius is a key parameter of the model.

Fig. ES $1 \mathrm{~b}$ and $\mathrm{c}$ show snapshots of the development of the model over time, for $r=5$; each individual imitated the vocalizations of its neighbours within a radius of 5. Acoustic attractors (Boeing, 2016) rapidly emerge across the colony after only a few iterations (electronic supplementary Animation ES 1), seen as contours of similar colours across the 2500 individuals. Over longer periods the contours of the 'soundscape' amalgamate and eventually converge onto a 
Modelling vocal imitation

steady state or uniform vocalization across the whole colony. If $r$ is smaller and if each individual learns only from some of its immediate neighbours, gradient patterns develop more slowly (Animation ES 2). If $r$ is larger, each individual learns from a larger fraction of the colony site, gradient patterns are lost sooner and the colony develops a uniform vocalization (Animation ES 3). If a small amount of noise is introduced into each vocalization at each iteration, representing movement within the colony or individual variation in learning, then the model never converges onto a steady state, and informative gradients and patterns persist across the colony indefinitely (Animation ES 4). The general behaviour of the model is robust for different values of $r$; attractors that have general utility only fail to emerge when each individual's learning is restricted to the same single neighbour for the life of the colony (Animation ES 5). At this stage, we are agnostic about any biological spatial or temporal scale, respectively for $r$ and for each time-step.

\section{What do the simulations mean?}

The series of simulations are intrinsically interesting, quite apart from any specific biological analogy. They show some of the behaviours that emerge from simply specified interactions among large numbers of computational addresses. Implementing the model and exploring it was a productive move.

Very many analogies could be proposed with different biological domains. The computational addresses could be claimed to be analogous to any organism — animal or plant. They could be analogous to different coherent groups of organisms. They could be analogous to individual cells, or to groups of cells. The information exchange could be analogous to sounds, to visual displays, to chemical transmitters, to small vibrations, and so on. All of these analogies could be more or less detailed, with more or less isomorphism between the model and the biological domain. Conventional computational modellers prefer the transparency of relatively simple models, 
Modelling vocal imitation

but our putative model is radically underspecified with respect to a very large range of potential biological domains.

We were led to consider the influence of the whole of the colony by moving towards greater biological specificity_harbour seals sometime live in colonies. However, this move to greater specificity was limited; we abstracted away everything from the real-world phenomenon except the exchange of information between statically connected individuals and their subsequent changes of state.

Critically, our attention had now turned in large part to the operation of the model, away from the real-world phenomenon. For instance, we ignored the real details of fundamental frequency, formant dispersion, and call duration; we reduced all three equally to variations in colour space. Further, we began exploring the effects of manipulating the parameters of the model. It is critical to re-engage with the potential biological domain; to move again to greater specificity, as opposed to seeing the biological domain through the lens of the model. The relation between the spontaneous materialism of working with the real biological phenomena and the process of theorizing can be addressed within a materialist framework, with respect to the related issues of abstraction and universals.

\section{Abstraction and one kind of universal}

We have identified the information changes in the model with vocal imitation in harbour seals, by one analogy among the many possible. Theorizing takes us back and forth between material entities and ideal entities. In computational modelling, the two interpenetrate in that the implemented model behaves materially in observable ways and everything hangs on the analogy between the biological phenomena and the implemented model. This relation has attracted increasing philosophical attention (e.g. Weisberg, 2012). 
Modelling vocal imitation

The materialist theory of knowledge has at its centre an approach to universals. The first kind of universal we need to understand is the abstract universal. Cognitive scientists typically understand a 'universal' to be something that unifies many things, something true of all minds, for instance, or of all languages. For biologists, the concept of universal applies to terms like 'species', 'predator' or 'prey', but arguably has rather less academic currency than in the study of cognition. Among biologists (as with most experimental scientists), there is a powerful, but typically implicit, convention of positivism - the philosophical position that there is a firm, known foundation (e.g. DNA) such that everything can be built up from that foundation, verifying each such addition, until the highest levels of biological organization (e.g. human cognition) are reached. Below, we take modern materialism to be the productive alternative to positivism in biological research.

An abstract universal is defined as something created to capture that which is similar across a number of different entities. Thus, 'verb' captures that which is similar across the words sneeze, inhabit, invoke, and so on; 'altruism' captures a range of behaviours that are claimed to be beneficial to the group but not (directly) to the individual; 'plant' captures that which is similar across trees, grasses, mosses and so on. Abstract universals are themselves relatively contentlessthey are the names of categories — and, critically, are always subject to defeat by new data. Thus, 'verb' does not stand up well in the analysis of the Salish languages (Kinkade, 1983); fungi are not straightforwardly plants (Martin, 1955). Abstract universals are nonetheless useful in getting initial traction on a particular domain, and in providing a vocabulary for research.

In theorizing, we typically consider ordered relations between abstract universals. In classic box-and-arrow models, psychologists specify such ordered relations between abstract universals (typically the labels on the boxes) and theorizing standardly consists of redefining those ordered relations. Memory research provides examples of such theorizing (e.g. Cowan, 2008). For instance, psychologists might divide processing into boxes labelled 'long term memory' and 'short term 
Modelling vocal imitation

memory', and might reorder the relations by inserting a new entity such as a 'buffer' or by pooling 'long term memory' and 'short term memory' into a single box representing a single process; the entities in the boxes are operationalized by laboratory procedures. Similarly, biologists may dispute the dividing line between natural selection and altruism, even to the point of eliminating altruism (e.g. Gilbert, 2010).

Levins (2006) explores abstraction in biological research, based on a typology developed by Ollman (2003): abstractions may be made from a particular vantage point within the domain, or on the basis of generalizations between different entities in the domain, or in terms of progression along some extended dimension in the domain. These abstractions are all seen as different ways of temporarily distorting the domain to reveal and bring into focus particular relations. The abstractions considered by Levins are all of the 'abstract universal' type. They give us a vocabulary with which to consider the phenomena under study, but they necessarily distort those same phenomena.

\section{The problem of defining the domain}

We now explore the relation between moving towards greater generality and greater specificity. We have identified the behaviour under investigation as 'vocal imitation'. According to Levins and Ollman, in using this term we have generalized in our abstraction (across vocalizing in different species). In our current materialist approach, we have created an abstract universal—a category that is recognized by biologists as capturing what is similar across different animal lineages generating new vocalizations influenced by those of conspecifics or other animals, or by environmental sounds (Janik \& Slater, 1997; Nottebohm, 1972).

This rare ability is distinct from 'auditory learning' or 'associative learning', as when a dog learns to respond to a spoken command by barking or some other behaviour. Biologists recognize it as occurring in only eight disparate animal lineages: parrots, elephants, bats, cetaceans, 
Modelling vocal imitation

hummingbirds, pinnipeds, songbirds, and humans. It invites interdisciplinary research. It has been studied for its neuroanatomical underpinnings (Bottjer, Halsema, Brown \& Miesner, 1989), its implications for phylogenesis (Nowicki \& Searcy, 2014) and ontogenesis (Marler, 1970), its place in ethology (Wright, Eberhard, Hobson, Avery \& Russello, 2010), and as a potential precursor for human speech (Teramitsu, Kudo, London, Geschwind \& White, 2004). The literature on vocal imitation contains numerous hypotheses addressing data within and across the eight lineages (Lattenkamp \& Vernes, 2018): effects of out-of-sight signaling (McComb, Moss, Sayialel \& Baker, 2000), sexual selection (Nowicki, Peters \& Podos, 1998), territory defence (Payne, 1981), individual (Janik, 2000) and group (Yurk, Barrett-Lennard, Ford \& Matkin, 2002) identification (Balsby, Momberg \& Dabelsteen, 2012), critical periods in development (Marler, 1970), information sharing (Fitch, 2004; Nowicki \& Searcy, 2014), geographic variation (Krebs \& Kroodsma, 1980), displays of dominance (Sanvito, Galimberti \& Miller, 2007), imitation of successful males (Sanvito, Galimberti \& Miller, 2007), vulnerability to predation (Igic, McLachlan, Lehtinen \& Magrath, 2015), adult tutelage (Phan, Pytte \& Vicario, 2006), indication of (brain) fitness (Catchpole, 1980), presence/absence of semantic information (Zann, 1996), copying of environmental sounds (Poole, Tyack, Stoeger-Horwath \& Watwood, 2005), fostering (Zann, 1985), domestication (Kagawa, Suzuki, Takahasi \& Okanoya, 2014), environmental change (Marler \& Peters, 1981), and the possibility of more limited forms of vocal imitation (Janik \& Slater, 1997).

The list above reflects the fact that biologists have worked with the abstract universal 'vocal imitation', exploring differing detailed behaviours in different species and different contexts. Our model at this stage putatively corresponds to harbour seals vocally imitating each other and potentially producing a complex soundscape. But what is the biological utility of this behaviour? Do we apply it to harbour seals, to elephants, to people? Without further content in the model, corresponding to some critical behaviour drawn from what we have seen is a bewildering list, all 
Modelling vocal imitation

that we have is an inherently interesting emergent behaviour of an abstract static crowd of individuals. The generalization implicit in 'vocal imitation' does not provide us with a research program to apply to a real biological domain.

We need to define a specific biological domain, but defining a domain is a notorious philosophical problem - the Frame Problem (Shanahan, 2016). However, a second type of universal provides us with a way forward.

\section{Abstraction and the second kind of universal}

A second, less well appreciated type of universal complements the abstract universal. It is the concrete universal. Consider the domain of human anatomy; how does the body work, how does it get to be the way it is? Probably the most influential entity in any determinate answer to those questions would be the stem cell. Given the necessary context, a stem cell can become any other type of cell in the body. In this sense, it is a universal-something that speaks to or unifies every other cell in the body. However, in other respects the stem cell, as a concrete universal, is unlike the abstract universal we considered above. A stem cell is itself a member of the set of cells, along with skin cells, neurons, lung cells, erythrocytes, and so on. It needed to be discovered. It is a material thing. There is no sense in which the entity 'stem cell' can be 'defeated' by new information.

'Explanation' within a materialist framework involves beginning with the totality of the domain and then abstracting away more and more (in the mind of the modeller) until there remains something that itself embodies the simplest example of the behaviour that characterizes the 'logic'

of the whole domain - the concrete universal. In our example, the stem cell materially embodies the processes of cellular functioning, cellular division, differentiation and migration. Once the concrete universal has been identified, the modeller is then able to take 'the return journey', adding back in more and more of the necessary context, producing more and more complexity, still mediated by that same concrete universal. The process of identifying the concrete universal is simultaneously 
Modelling vocal imitation

one of defining the domain of interest. The fullest understanding of the domain is available when the modeller is able to move fluidly 'upwards' or 'downwards' at any point between the totality of the domain and the material entity that has been identified as the concrete universal. For researchers in the conventional modelling paradigm with its emphasis on simplicity, the critical point is that the continuing role of the concrete universal in the return trip to complexity means a simultaneous, seemingly paradoxical increase in parsimony - everything is now seen in the terms dictated by the concrete universal. (For a review of the history of the concrete universal, from Vico to Ilyenkov, see Shillcock, 2014.)

\section{Defining a biological domain for productive research}

We had reached, above, a dilemma in defining the biological domain that most productively 'fits' the generation of attractors in a static crowd of individuals imitating each other's 'vocalizations'. The abstract universal 'vocal imitation' revealed a sprawling list of studies involving many animals, many behaviours and many other factors. In each such study, researchers explored what was seen as vocal imitation in a particular biological context, but little if any content can be added to the abstract universal 'vocal imitation' itself. Its synchronic limits are easily reached by counterexamples in which vocal imitation is used: for example, for displays of dominance in one species but not in another. Its diachronic limits are reached by the fact that any claim regarding a common evolutionary origin of vocal imitation is severely underdetermined by the data; for instance, humans and hummingbirds both exhibit vocal imitation-convergent evolution is more likely than a common origin.

The way out of the impasse is by a process of accommodation between the abstract and the concrete. We can move 'horizontally' across the phenomena covered by the abstract universal 'vocal imitation', such that we assess the different explorations of that entity in the list above. We move from species to species, from one claimed function of vocal imitation to another. In 
Modelling vocal imitation

considering each, we move 'downwards' into the material detail being considered, with the goal of identifying a concrete universal within that particular domain, meaning that we simultaneously identify a domain — with its own way of behaving, its own 'logic' — and some material process that characterizes that logic. We thus explore the concrete (i.e. densely interconnected, in materialist terms) biological world guided by the abstract universal, vocal imitation.

At the same time as we try to 'fit' the biological world to our model, we try to fit the model to the biological data being encountered. We have seen that the model domain is very sparsely specified (i.e. relatively 'abstract' in materialist terms). The process of exploring different possible models is exactly the same as with exploring different biological data: the abstract universal 'computational model' refers to a set of different entities — different computational models with different architectures, different behaviours, directed at different phenomena-each of which can be assessed for ways of modifying the existing model so as to fit the biological data.

The dialectic between the biological domain and the model domain is negotiated by the researcher in a seemingly idiosyncratic manner (although heuristics could surely be proposed). The criterion for success is that relatively isomorphic processes are identified in the two domains, with those processes corresponding to a good candidate for a concrete universal in the biological domain. In short, the concrete universal will have mediated the process of accommodation between the two material domains, biology and implemented computation.

In our case study, the model created an attractor-based 'soundscape' across the colony of vocal imitators, but it had no apparent function. We needed to abstract away from the rich biological data so that it constituted a system with its own essential logic. The relevant concrete universal will be something material. In the materialist approach the researcher is more concerned with change, with a world of processes rather than a world of entities. What processes occur in colonies of the kinds created by some of the eight lineages that exhibit vocal imitation? 
Modelling vocal imitation

Can 'nurture' be the relevant concrete universal? 'Nurture' is an abstract universal capturing that which is common between suckling, providing captured food, keeping warm, protecting from predators, cleaning, grooming, giving emotional and physical support, and so on. The simplest, basic, material process that is essential to all of these activities is the process of the parent identifying the infant and moving closer to provide nurture. This process is critical to the colony's existence and in this sense mediates every other process in the colony.

We now add parents to the model.

\section{Adding parental navigation to the model}

We hypothesize that the contours and gradients of the biological colony's attractor-based soundscape help returning parents to find their offspring. To test this prediction in the model, we selected infants at random and required parents to start at the edge of the colony and find their infant. (Simulations in which parents started at non-edge random points behaved similarly.) The simulations occurred over static snapshots of the colony's soundscape, not the evolving soundscape (we are still agnostic about real temporal and spatial scale). The parent could move to a different square at each step of the simulation. The simulation terminated when the parent found the infant, or after 1000 steps. Parents were given three search strategies: a baseline simple random walk, a Lévy flight and a gradient ascent. These strategies were based on biological observations made across different species.

We simulated each of the search strategies 10,000 times. We calculated the mean number of steps required to find the infant for each strategy, which we compared using two-tailed $t$-tests.

(1) Simple random walk. The parent makes the next move randomly to one of the 8 adjacent squares. We found that the parent typically explored a small region fairly exhaustively and failed if the infant was not in that region (electronic supplementary Fig. 1a and Animation ES 6). 
Modelling vocal imitation

(2) Lévy flight. The parent takes a random walk and draws the size of each move from a (heavytailed) power law distribution. A Lévy flight search pattern may be advantageous for foraging in sparsely distributed search sites (Bartumeus, Catalan, Fulco, Lyra \& Viswanathan, 2002;

Bartumeus, Da Luz, Viswanathan \& Catalan, 2005; Viswanathan, et al., 1999), as with albatrosses (Viswanathan et al., 1996) and spider-monkeys (Ramos-Fernández et al., 2004). We found that the parent explored more regions, crisscrossing the colony when the movement distance was picked from the heavy tail (Fig. 1b), although the strategy still often failed (Animation ES 7). Despite claims of Lévy flight superiority in the optimal foraging literature, we found no significant difference from a simple random walk $(t=0.082, \mathrm{p}=0.93$, n.s. $)$.

(3) Gradient ascent. The parent follows a simple hill-climbing algorithm. At each move it considers the immediately adjacent squares and moves in the direction most similar to the infant's vocalization. If the parent reaches a local minimum in the gradient without finding the infant—such that each adjacent square is less similar than the current one - a direction is chosen at random. We found that the parent often picked direct and successful routes to the infant (Fig. 1c and Animations ES 8-10), although it could fail if the initialization placed the infant beyond a significant barrier of dissimilar sounds. The gradient ascent strategy significantly outperformed the simple random walk $(t=106.0, \mathrm{p}<0.001)$ and the Lévy flight $(t=106.5, \mathrm{p}<0.001)$.

At this point we have discovered two further intrinsically interesting facts about 'vocal imitation' in a large static crowd. First, the complex attractor structure can be adaptive, facilitating a returning parent finding their progeny. Second, the attractor-based guidance is robust and improves across time; Fig. 2 shows for each strategy the mean number of steps required to reach the infant at successive 10-epoch developments of the colony. The gradient ascent strategy achieves a rapid superiority that increases and is sustained as the attractor structure becomes simpler over time. 
Modelling vocal imitation

\section{Making the biological system more specific}

Biologists have studied our abstract universal, vocal imitation, in relation to many different factors. Each case could be seen as a biological domain to be modelled. We restricted ourselves to those domains involving large breeding colonies. We further specified that parental navigation in the colony might be a viable concrete universal. In considering the navigation strategy, we even explored the different biologically observed navigation strategies covered by the abstract universal 'navigation strategy', some of which might not have even been demonstrated to exist in the lineages that exhibit vocal imitation.

What we were doing here was negotiating the dialectic between the abstract and the concrete, using the abstract universal to jump from case to case before exploring the biological details of that case, searching for a plausible concrete universal, something that embodies the 'logic' of the biological domain but something that also is isomorphic with some important material behaviour of the computational model.

We now make the important move- based on this mutual accommodation between the modelling domain and the biological domain - to concentrate on bats as the best fit with the emergent behaviour of the model. The biological domain of the harbour seal no longer matched the putative maternal behaviour explored in the model: harbour seal pups can swim from birth and follow their mother into the water, thus contradicting our assumptions about movement and nurture in colonies. Even here, though, we have leapfrogged the generic 'bats' as mentioned in introductions to vocal imitation research and we are directly led to consider the particular bat species that have been studied.

Esser (1994) presented the first clear evidence for vocal imitation in a terrestrial non-human animal, Phyllostomus discolor, the lesser spear-nosed bat (cf. Esser \& Schubert, 1998). Pups progressively adapt their isolation calls by audio-vocal learning to the individually distinct 
Modelling vocal imitation

frequency modulation pattern of the maternal directive call. The vocal signature consists of the number of frequency minima and maxima per call, the carrier frequency, and the modulation frequency.

Consider the Mexican free-tailed bat, Tadarida brasiliensis mexicana. It roosts in very large colonies of $20 \mathrm{M}+$ in dark caves, with nursery regions of the cave being populated with $300-500$ pups per square foot. Mothers leave pups in large creches and return two or more time daily to nurse, for a period of weeks, making location of a mother's own pup a prodigious, central problem to be solved (McCracken \& Gustin, 1991) and matching our putative concrete universal for the domain to be modelled. McCracken (1984) shows that nursing in this species is not indiscriminate, but that at any one time some $17 \%$ of mothers may be nursing a genetically unrelated pup; what is the evolutionary origin of such alloparental care? Gelfand and McCracken (1986) demonstrate individual variation in pups' calls and show that the calls change over time. Use of multiple sensory cues is to be expected: mothers use location information to focus the search for their pups (McCracken 1993), produce individually distinctive directive calls that elicit calling by pups (Balcombe \& McCracken 1992), and then combine olfactory and individually distinctive vocal cues to identify their pup (Gustin \& McCracken 1987; Balcombe 1990).

McCracken and Gustin (1991) review the claim that there is a thermal benefit from aggregating in large populations; temperatures of 35-40 degrees $\mathrm{C}$ are observed. They leave open the possibility of other benefits. Our modelling suggests that locating an infant is easier when that infant can imitate many other infants, necessitating close aggregation.

Research on the spear-nosed bat, Phyllostomus hastatus, has also involved the role of vocalization in uniting nursing mothers and pups in dark cave environments. Each night, all but a few adults depart to forage, leaving pups behind in site-specific roosts. Their groups number eight to 40 individuals (McCracken \& Bradbury 1981; McCracken 1987). Females within a group give 
Modelling vocal imitation

birth synchronously (Porter \& Wilkinson 2001), the first flight by the pup occurring after 6 weeks (Stern \& Kunz 1998). In the context of a dark cave, females first rely on pup vocalizations — 'isolation calls' — to find fallen pups (Gould et al. 1973). These calls are also used in recognition; they carry individually distinctive frequency spectra, and psychophysical studies have shown that $P$. hastatus can discriminate between isolation calls from different pups in the same social group (Bohn et al. 2007).

As this partial review of the literature shows, bats represent a biological domain that productively fits the emergent behaviours of the model at this point. We could make the biological domain even more concrete by settling exclusively on the Mexican free-tailed bat and requiring results obtained with other bat species to be replicated with that species.

As the mother physically contacts the pup, the engagement changes qualitatively into other behaviours (feeding, grooming, and so on) and the relevant context — the domain — changes too. We may well need to specify a different concrete universal at this point as we focus in on, for instance, how the mother feeds the pup.

\section{Where does the modelling lead?}

In both materialist and conventional approaches to modelling, the model is a tool for research. The model succeeds if it drives further empirical research in the biological domain. However, in the materialist approach, the goal is always the return trip to the biological totality of the domain being studied - to be understood in terms of the concrete universal. In conventional approaches, the goal is typically the simplicity of the successful model, leading to the model-asexplanation and the ensuing focus on the philosophical status of modelling. The most satisfactory provisional conclusion from the modelling is a set of further developments for the model and a set of further questions regarding the biological domain. 
Modelling vocal imitation

In our model so far, each node represents a bat pup. To match the biological data better, we may decide that some of the nodes represent mothers. We may further change the proportion of the time that mothers are present. In short, the model may be further accommodated to the now more specific biological data.

In the biological domain of Tadarida brasiliensis mexicana we can ask what is the relation between the pup and its mother? Our modelling suggests that the desired parenting effects emerge, with maximum parsimony, without specifying a bidirectional exclusive relationship; the pup need simply imitate any vocalizations in proportion to proximity and probability of exposure. Indeed, our model shows that informative attractors fail to emerge when imitation is restricted to single adjacent pairs; counterintuitively, an exclusive mother-pup bond may be counter-productive.

We can ask does the presence of informative auditory attractors mediate the visiting of nongenetically related pups? Alloparental care may be an emergent effect of a mother navigating the attractor structure in a timed way or with less than 100\% accuracy, resulting in (as observed by McCracken, 1984) a tolerable $17 \%$ of alloparental nurture. The apparent altruism of alloparental care may emerge from very parsimonious navigation parameters (although this origin does not prevent the behaviours being themselves the origin of further behaviours that take the apparently altruistic behaviour 'at face value').

We can ask what dimensions of the vocalization are imitated and how fast? In our modelling we have not been specific about absolute time. We need to assume that a pup's vocalization changes fast enough to generate informative attractors but not so much that the mother completely fails to recognize the pup on returning from foraging. Aspects of the vocalization, such as base frequency, frequency modulation and call duration, may all change independently and may be differentially informative. 
Modelling vocal imitation

We can ask what is the relationship between infant vocal imitation and wider adult behaviour such as foraging? Bohn, Moss and Wilkinson (2009) suggest that alloparental care is not directly explained by kinship or direct/generalized reciprocity but by a complex interplay between group membership and cooperative foraging. Social groups forage together using learned groupspecific vocalizations (Boughman 1998; Wilkinson \& Boughman 1998). We should expect continuities between a behaviour central enough to be a candidate concrete universal in one domain (navigation in nurture) and similar behaviours in related domains (navigation in foraging). We may expect infant communication to have implications for adult vocalizations. How do acoustic attractor structures develop across real colonies? Does a whole adult group adopting a single particular vocalization (Boughman, 1998) reflect the final converged destination of the developing attractor structure?

We can ask whether we can analogously model behaviours related to geography and territory in adult animals that exhibit vocal imitation but do not nurture their young in large colonies. We can ask how vocal imitation can be retained beyond any critical period and into adulthood (Brenowitz \& Beecher, 2005; Tyack, 2008) to then be exapted to new social functions (Janik \& Slater, 2000).

We have provided a parsimonious account of the role of vocal imitation in one species of bat. Assuming convergent evolution of vocal imitation (cf. Knörnschild, Nagy, Metz, Mayer \& von Helversen, 2009), we can ask whether some of the same evolutionary pressures produce vocal imitation in other lineages.

The suggestions, above, for developing the model, together with the list of further questions about the biological domain are enough to demonstrate the productivity both of the materialist approach and of our specific model of vocal imitation in bats.

\section{What is the relation between the biological system and the model system?}


Modelling vocal imitation

A materialist ontology 'grounds out' with various categories such as individual, general, difference, form, content, appearance, cause, particular, contradiction, universal, abstract, concrete, simplicity, complexity, and so on, and the relations between them. Thus, for instance, we can see the biological domain and the model domain as equally material entities on a dimension between the individual and the general. Nothing is completely individual; it will always share certain similarities with other entities. Nothing is completely general; it will always be distinct from everything else in some way. The two domains are at different points on the continuum, with implications for the question of identifying isomorphisms between the two. Similarly, we can locate the two domains on the dimension between content and form, and between complexity and simplicity. The biological domain is located towards the complex, contentful, concrete (i.e. highly interconnected) and individual ends of the relevant dimensions. The domain of the implemented computational model is located towards the simple, formal, abstract (i.e. sparsely interconnected) and general ends of the relevant dimensions.

Consider the relation between a large bat colony and a smaller one. The latter is trivially a 'model' of the former, being more tractable for the researcher in various ways. If we keep abstracting material detail away from the smaller domain, the trajectory of abstraction that does least damage to the relation with the large colony and most reveals the nature of the large colony is one that ends with the concrete universal - the material characterization of the 'logic' of the colony. In our case study of vocal imitation in a bat colony, we have identified the concrete universal as the process of the mother orienting to its pup. The concrete universal is a material entity but it is still an abstraction from the large colony. Certain aspects of the totality of the colony have retreated to the background: the exact diet of the mother, the mother's foraging behaviours, the precise flight behaviours of the mother, the activity of the pup's father, the behaviour of the mother after 
Modelling vocal imitation

contacting the pup, and so on. What emerges is a view of the large colony that is more clearly a system — one that is mediated by the material concrete universal.

In the materialist approach, the modelling process is a relation between two domains.

Modellers conventionally understand the asymmetry between the two domains as the biological domain model being the starting point and the modelling domain being the goal, with the model representing biological processing in the simplest terms. Simplicity is typically an explicit goal, to the point that the model is seen as an explanation of the real-world phenomenon. Although perhaps most modellers subscribe to the goal of a virtuous spiral between empirical research involving the real-world phenomenon and simulations with the computational model, the reality may be that such spirals are more limited than we suspect. Making the architecture of a computational model more elaborate is conventionally seen as contradicting the principle goal of modelling — simplicity. Chawla and Shillcock (2019) take a test case of a high-profile cognitive model and show that its citations overwhelmingly refer to the success of the model's architecture or to its algorithmic content, as opposed to elaboration of the model's architecture. There are, naturally, implemented models that have generated long-running, productive research programs, but even this outcome is not the same as the prospect of an ongoing exploration of open problems related to the original behaviour and leading back to the biological totality of the domain. One outcome is that interest in conventional modelling has become more focused on the abstract philosophical nature of the relation between data and model, as opposed to seeing the model as a tool for researching the biological domain.

We have argued that the relation between the biological domain and the model domain develops through mutual accommodation. We explore the biological domain, mediated by the abstract universal — vocal imitation, in our test case. This exploration may be unreported, implicit in the researcher's choice of modelling problem. It may be implicit in the choice of a simplified 
Modelling vocal imitation

problem, as when, for instance, McClelland and Rumelhart (1981) restrict the domain of the Interactive-Activation Model (IAM) of word recognition to the processing of four-letter words. The IAM has contributed enormously to research, prompting experiments with human participants, creating a taxonomy of effects in single-word reading, and being incorporated in one of the most influential models of lexical processing, the Dual-Route Cascaded model (Coltheart, Rastle, Perry, Langdon \& Ziegler, 2001). Its restriction to four-letter words is generally seen as a necessary means of focusing on the problem and as an attractive simplification, despite the fact that extending the model to words of variable length raises central problems for the logic of its processing and has not been attempted to date.

We explore the model domain and its analogy to the biological domain, mediated by the concrete universal. The biological domain is framed — turned into a system — by including detail that is mediated by the concrete universal. Any factor that the concrete universal speaks to is relevant to the model and may be incorporated into the model. Here we see a major difference between conventional modelling and materialist modelling. In conventional modelling, there is a one-way direction of travel towards simplicity. In materialist modelling the researcher attempts the return trip towards understanding the full complexity of the biological domain, incorporating increasing detail into the model. In the materialist model the concrete universal has already materially embodied the internal 'logic' of the biological domain. Further detail paradoxically makes the model more parsimonious as everything relates to the concrete universal.

\section{Conclusions}

We have presented a model of the critical interaction between bat mothers and pups in large colonies, with particular reference to the Mexican free-tailed bat, Tadarida brasiliensis mexicana. Unusually, we have described the full range of steps we took-from harbour seals to bats-in arriving at our model. We have been explicit about employing a materialist theory of knowledge, 
Modelling vocal imitation

with its crucial theory of abstraction and its concepts of concrete and abstract universals. Three conclusions emerge. (1) A productive and developing implemented computational model that generates a specific program of biological research questions. (2) A theory of the relation between the two material domains - biological behaviours and implemented computational model. (3) A critique of conventional computational modelling.

There is no philosophical short-cut to an effective computational model. Knowledge of the primary biological data is vital, as is knowledge of the computational-modelling options. However, an explicit theory of knowledge can unpack modelling intuitions and can assist in steering through the huge many-to-many ambiguity between the biological data and the modelling options. A materialist theory of knowledge has served this purpose in the current case study. 
Modelling vocal imitation

Fig. 1. Examples of a parent searching for its infant, using the respective search strategies. (a)

Simple random walk; (b) Lévy flight; (c) Gradient ascent.

(a)

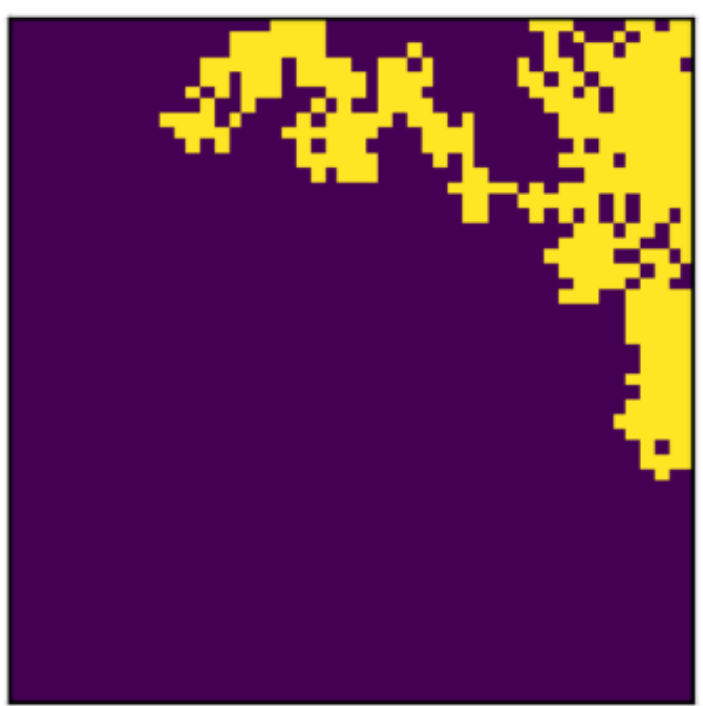

(b)

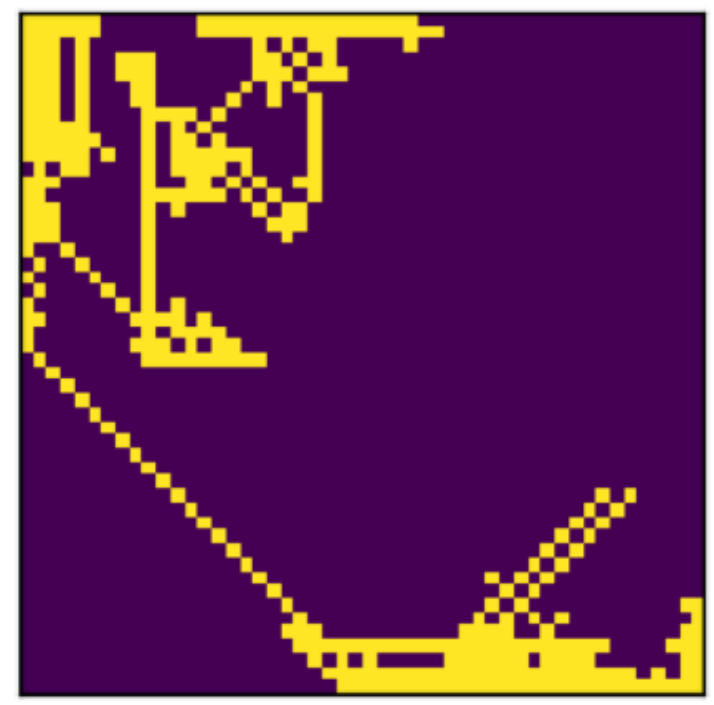

(c)

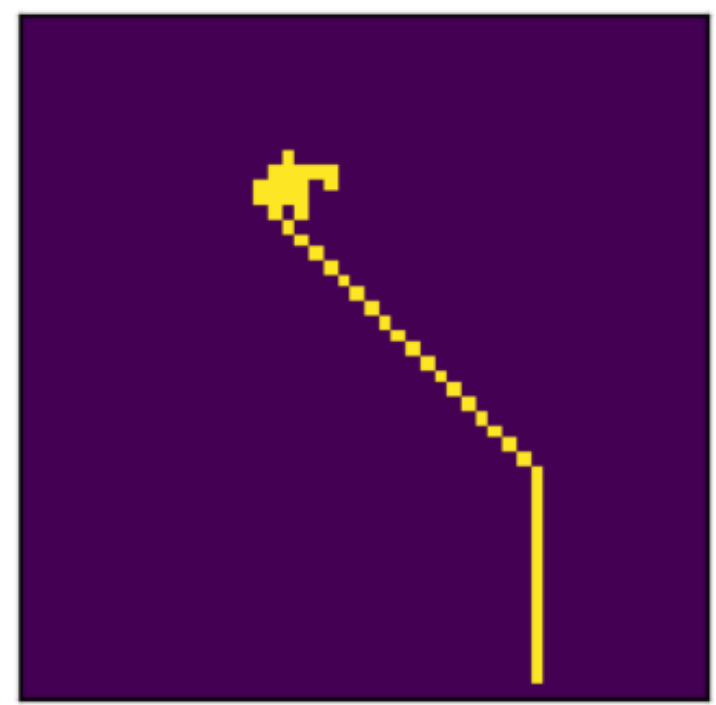


Modelling vocal imitation

Fig. 2. Comparison of the three strategies of random walk, Lévy flight and gradient ascent. Means of 10,000 trials are plotted for each strategy every 10 epochs for the evolving attractor structure, showing the mean number of steps required to reach the infant.

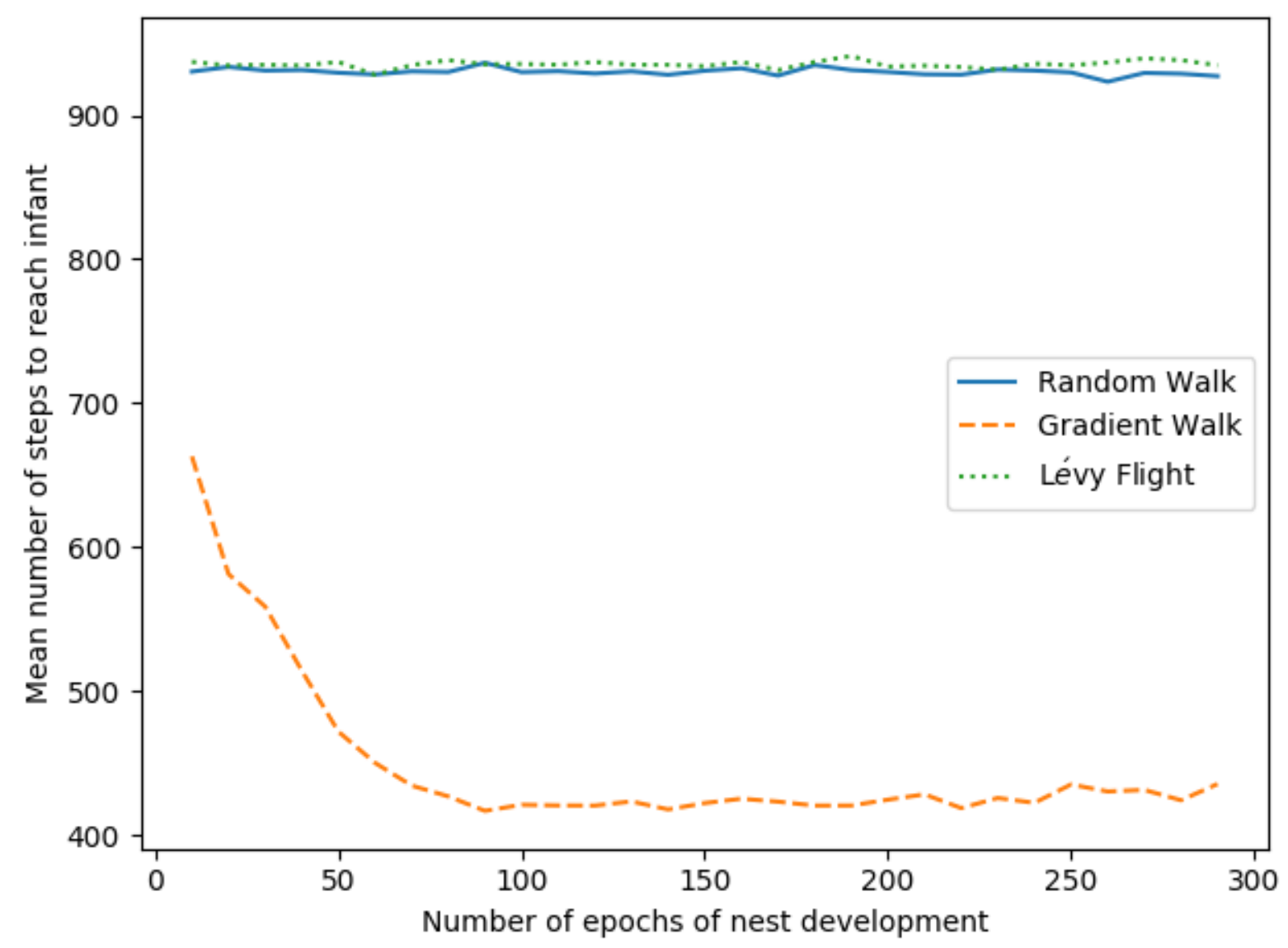




\section{References}

Balcombe, J. P., \& McCracken, G. F. (1992). Vocal recognition in Mexican free-tailed bats: do pups recognize mothers?. Animal Behaviour, 43(1), 79-87.

Bartumeus, F., Catalan, J., Fulco, U., Lyra, M. \& Viswanathan, G. Optimizing the encounter rate in biological interactions: Lévy versus Brownian strategies. Physical Review Letters 88, 097901 (2002).

Bartumeus, F., Da Luz, M. E., Viswanathan, G. \& Catalan, J. Animal search strategies: a quantitative random-walk analysis. Ecology 86, 3078-3087 (2005).

Boeing, G. Visual analysis of nonlinear dynamical systems: Chaos, fractals, self-similarity and the limits of prediction. Systems 4, 37 (2016).

Bohn, K. M., Moss, C. F., \& Wilkinson, G. S. (2009). Pup guarding by greater spear-nosed bats. Behavioral Ecology and Sociobiology, 63(12), 1693-1703.

Boughman, J. W. Vocal learning by greater spear-nosed bats. Proceedings of the Royal Society of London B: Biological Sciences 265, 227-233 (1998).

Bottjer, S. W., Halsema, K. A., Brown, S. A. \& Miesner, E. A. (1989). Axonal connections of a forebrain nucleus involved with vocal learning in zebra finches. Journal of Comparative Neurology 279, 312-326 (1989).

Brenowitz, E. A. \& Beecher, M. D. Song learning in birds: diversity and plasticity, opportunities and challenges. Trends in Neurosciences 28, 127-132 (2005).

Bullinaria, J. A. (2009). Lifetime learning as a factor in life history evolution. Artificial Life, 15(4), $389-409$

Catchpole, C.K. (1980): Sexual selection and the evolution of complex songs among European warblers of the genus Acrocephalus. Behaviour 74: 149 \pm 166 . 
Chawla, M., \& Shillcock, R. (2019, February 7). What is the role of computational models in Cognitive Science? A quantitative and qualitative analysis of the history of the TRACE model of speech segmentation. Retrieved from psyarxiv.com $/ \mathrm{m} 79 \mathrm{fw}$

Coltheart, M., Rastle, K., Perry, C., Langdon, R., \& Ziegler, J. (2001). DRC: a dual route cascaded model of visual word recognition and reading aloud. Psychological Review, 108(1), 204.

Esser, K. H. (1994). Audio-vocal learning in a non-human mammal: the lesser spear-nosed bat Phyllostomus discolor. Neuroreport, 5(14), 1718-1720

Esser, K. H., \& Schubert, J. (1998). Vocal dialects in the lesser spear-nosed bat Phyllostomus discolor. Naturwissenschaften, 85(7), 347-349.

Fitch, W. T. (2004). Kin selection and 'mother tongues': a neglected component in language evolution. Evolution of communication systems: A comparative approach, 275-296.

Gelfand, D. L., \& McCracken, G. F. (1986). Individual variation in the isolation calls of Mexican free-tailed bat pups (Tadarida brasiliensis mexicana). Animal Behaviour, 34(4), 1078-1086.

Igic, B., McLachlan, J., Lehtinen, I., \& Magrath, R. D. (2015). Crying wolf to a predator: deceptive vocal mimicry by a bird protecting young. Proc. R. Soc. B, 282(1809), 20150798.

Janik, V. M. (2000). Whistle matching in wild bottlenose dolphins (Tursiops truncatus). Science $289,1355-1357$.

Janik, V. M., \& Slater, P. J. (1997). Vocal learning in mammals. Advances in the Study of Behaviour, $26,59-100$.

Janik, V. M. \& Slater, P. J. The different roles of social learning in vocal communication. Animal Behaviour 60, 1-11 (2000).

Kagawa, H., Suzuki, K., Takahasi, M., \& Okanoya, K. (2014). Domestication changes innate constraints for birdsong learning. Behavioural Processes, 106, 91-97.

Kinkade, M. D. (1983). Salish evidence against the universality of 'noun' and 'verb'. Lingua, 60(1), 25-39. 
Knörnschild, M., Nagy, M., Metz, M., Mayer, F., \& von Helversen, O. (2009). Complex vocal imitation during ontogeny in a bat. Biology Letters, rsbl20090685.

Krebs, J. R. \& Kroodsma, D. E. (1980). In Advances in the Study of Behavior Vol. 11 143-177 (Elsevier,).

Lattenkamp, E. Z. \& Vernes, S. C. (2018). Vocal learning: a language-relevant trait in need of a broad cross-species approach. Current Opinion in Behavioral Sciences.

Marler, P. (1970). A comparative approach to vocal learning: song development in White-crowned Sparrows. Journal of Comparative and Physiological Psychology 71, 1.

Marler, P., \& Peters, S. (1981). Birdsong and speech: Evidence for special processing. Perspectives on the Study of Speech, 75-112.

Martin, G. W. (1955). Are fungi plants?. Mycologia, 47(6), 779-792.

McClelland, J. L. The place of modeling in cognitive science. Topics in Cognitive Science 1, 11-38 (2009).

McClelland, J. L., \& Rumelhart, D. E. (1981). An interactive activation model of context effects in letter perception: I. An account of basic findings. Psychological Review, 88(5), 375.

McCracken, G. F. (1984). Communal nursing in Mexican free-tailed bat maternity colonies. Science, 223(4640), 1090-1091.

McCracken, G. F., \& Gustin, M. K. (1991). Nursing behavior in Mexican free-tailed bat maternity colonies. Ethology, 89(4), 305-321.

McComb, K., Moss, C., Sayialel, S. \& Baker, L. (2000). Unusually extensive networks of vocal recognition in African elephants. Animal Behaviour 59, 1103-1109.

Nottebohm, F. (1972). The origins of vocal learning. The American NaturalistI 106, 116-140.

Nowicki, S. \& Searcy, W. A. (2014). The evolution of vocal learning. Curr Opin Neurobiol 28, $48-53$. 
Nowicki, S., Peters, S. \& Podos, J. (1998). Song learning, early nutrition and sexual selection in songbirds. American Zoologist 38, 179-190.

Ollman, B. (2003). Dance of the dialectic: steps in Marx's method. University of Illinois Press, Chicago.

Payne, R. B. (1981). Song learning and social interaction in indigo buntings. Animal Behaviour 29, 688-697.

Phan, M. L., Pytte, C. L., \& Vicario, D. S. (2006). Early auditory experience generates long-lasting memories that may subserve vocal learning in songbirds. Proceedings of the National Academy of Sciences, 103(4), 1088-1093.

Ramos-Fernández, G. et al. Lévy walk patterns in the foraging movements of spider monkeys (Ateles geoffroyi). Behavioral ecology and Sociobiology 55, 223-230 (2004).

Reynolds, C. (1987). Flocks, herds and schools: A distributed behavioral model. SIGGRAPH '87: Proceedings of the 14th Annual Conference on Computer Graphics and Interactive Techniques. Association for Computing Machinery. pp. 25-34.

Sanvito, S., Galimberti, F., \& Miller, E. H. (2007). Observational evidences of vocal learning in southern elephant seals: a longitudinal study. Ethology, 113(2), 137-146.

Shanahan, Murray, "The Frame Problem", The Stanford Encyclopedia of Philosophy (Spring 2016 Edition), Edward N. Zalta (ed.), URL = <https://plato.stanford.edu/archives/spr2016/entries/ frame-problem/>.

Shillcock, R. (2014). The Concrete Universal and Cognitive Science. Axiomathes, 24(1), 63-80.

Teramitsu, I., Kudo, L. C., London, S. E., Geschwind, D. H. \& White, S. A. (2004). Parallel FoxP1 and FoxP2 expression in songbird and human brain predicts functional interaction. Journal of Neuroscience 24, 3152-3163. 
Tyack, P. L. Convergence of calls as animals form social bonds, active compensation for noisy communication channels, and the evolution of vocal learning in mammals. Journal of Comparative Psychology 122, 319 (2008).

Viswanathan, G. M. et al. Optimizing the success of random searches. Nature 401, 911 (1999).

Viswanathan, G. M. et al. Lévy flight search patterns of wandering albatrosses. Nature 381, 413 (1996).

Weisberg, M. (2012). Simulation and similarity: Using models to understand the world. Oxford University Press.

Wright, T. F., Eberhard, J., Hobson, E., Avery, M. L. \& Russello, M. (2010). Behavioral flexibility and species invasions: the adaptive flexibility hypothesis. Ethology Ecology \& Evolution 22, $393-404$.

Yurk, H., Barrett-Lennard, L., Ford, J. \& Matkin, C. (2002). Cultural transmission within maternal lineages: vocal clans in resident killer whales in southern Alaska. Animal Behaviour 63, 1103-1119.

Zann, R. (1985). Ontogeny of the zebra finch distance call: I. Effects of cross-fostering to Bengalese finches. Zeitschrift für Tierpsychologie, 68(1), 1-23.

Zann RA (1996) The Zebra Finch: a synthesis of field and laboratory studies. Oxford Univ Press, New York. 
Acknowledgements

\section{Author contributions}

R.S. conceived the study; B.M., R.S. and A.R developed the research; B.M performed the simulations; R.S.,

B.M. and A.R. wrote the paper. All authors gave final approval for publication.

\section{Data accessibility}

The modelling code is available on GitHub:

https://github.com/Bmillidgework/Vocal_Learning

\section{Competing interests}

We have no competing interests.

\section{Ethical statement}

The research had no ethical implications. 